\title{
Interplay between human cytomegalovirus and dendritic cells in $T$ cell activation
}

\author{
Hélène Martin · Marie Mandron · Christian Davrinche
}

Received: 3 December 2007 / Published online: 9 February 2008

(C) Springer-Verlag 2008

\begin{abstract}
Control of human cytomegalovirus (HCMV) infection and prevention of associated diseases in immunocompetent hosts are ensured mainly by CD8+ T cells, in spite of numerous viral tricks to impair antigen presentation and activation of $\mathrm{T}$ cells. At sites of primary infection, dendritic cells (DCs) are in the forefront to ensure capture of viral antigens and their capacity to bypass the effects of viral immunoevasins is crucial in moulding CD8+ $\mathrm{T}$ cell repertoire. In HCMV-seropositive donors, the spectrum of CD8+ $\mathrm{T}$ cells specificities was shown to include immediate-early (IE), early (E) and late (L) gene products, a surprising finding if we consider that expression of immunoevasins could paralyse infected DCs from the IE phase of infection. In the present report, we suggest that uninfected dendritic cells could acquire HCMV-antigens derived from input virus or neosynthesis, either in soluble forms or in association with infected dead cells resulting from death-ligand-mediated apoptosis and necrosis. Activation of naïve CD8+ T cells could then occur in lymph nodes through cross-presentation by antigen-loaded DCs, providing an explanation for shape and size of the memory compartment.
\end{abstract}

Keywords Cytomegalovirus · Dendritic cell · Cross-presentation $\cdot \mathrm{T}$ cell repertoire

\section{Introduction}

Clearing of a viral infection depends mostly on the ability of the host to mount an efficient primary anti-viral

H. Martin · M. Mandron · C. Davrinche $(\bowtie)$ Centre de Physiopathologie de Toulouse Purpan, INSERM, U563, Toulouse 31300, France e-mail: davrinch@toulouse.inserm.fr response and to get it in memory to overcome future viral challenge. In this process, dendritic cells (DCs) are crucial as they are sentinels located in most organs to monitor entry of viruses and initiate activation and proliferation of anti-viral T cells in lymph nodes. Two subsets of dendritic cells, plasmacytoids and myeloids, are now considered to play a role in innate as well as in adaptive response against viruses through both secretion of anti-viral type I interferons and activation of specific naïve $\mathrm{T}$ cells. Because of their vital role in inducing potent anti-viral immune responses, dendritic cells are major target for viruses and viral products which aim at blocking their function at any stage of antigen presentation, migration to secondary lymphoid organs and activation of $\mathrm{T}$ cells. Moreover, some viruses such as HIV and human cytomegalovirus (HCMV) use the C-type lectin receptor DC-SIGN on dendritic cells as a vector to ensure their transmission to other cells either in the periphery or in lymph nodes [1,2]. Direct arguments that DCs are real targets for HCMV in vivo are suggested, for instance, by the ability of the virus to infect hematopoietic progenitor cells [3] and by the presence of viral DNA in purified DCs from viremic renal transplant patients as well as from healthy virus carriers $[4,5]$. HCMV is a latent herpesvirus, which can be considered as a spearhead in exploiting co-existence with the host to develop numerous immunoevasion mechanisms, and virus-encoded immunoevasins have been described that impair many of dendritic cells functions including antigen presentation to CD4+ and $\mathrm{CD} 8+\mathrm{T}$ cells [6]. Even though CD4+ T cells play a role in mounting anti-viral response, cytotoxic CD8+ $\mathrm{T}$ lymphocytes (CTL) are considered as the main effectors involved in protection against CMV-associated diseases, as demonstrated by in vivo adoptive transfer in mouse infected with mouse CMV (MCMV) [7] and in bone marrow transplanted humans $[8,9]$. CTL are supposed to exert their 
anti-viral activity on target cells to ensure protection of the host against HCMV, either in primary infection or reinfection or reactivation of the latent virus. This raises the question of how a memory $\mathrm{T}$ cell repertoire against HCMV could be moulded in conditions where DCs are supposed to be paralysed by immunoevasins, and how effector CTL could ensure eradication of infected cells in tissues after re-infection or reactivation. Surprisingly, the CTL repertoire against HCMV appeared to exhibit broader specificities than expected, including proteins available at any stage of the virus cycle (immediate-early IE, early E, late L) and the question takes even more sense with respect to the fact that immunoevasins could be produced in the IE phase of infection. In response to this question, we can imagine multiple scenarios where the kinetics of events takes a major part including (1) time points at which immunoevasins are produced in infected cells and how efficient they are with respect to MHC polymorphism, (2) HCMV strains variability and virulence and (3) how long it takes for cells to produce new virions, for antigen-loaded DCs to reach lymph nodes and for activated CTL to migrate back to the site of infection where they could encounter newly infected targets.

Besides these possible scenarios, cross-presentation of HCMV antigens by uninfected DCs to CD8+ T cells could be considered as a key process in initiating CTL response provided that viral proteins are made available for ingestion and processing by DCs and that they can be transported to lymph nodes for activation of naïve $\mathrm{T}$ cells. All of these points will be discussed throughout the present paper.

\section{Alteration in dendritic cells functions by HCMV}

Innate anti-viral response

Dendritic cells are crucial in host innate defence since they are in the first line of virus sensing at primary sites of infection through binding to pathogen recognition receptors (PRR) such as toll-like receptors (TLR), expressed either at the cell surface or in cell compartments (for review see [10]). Interactions between the envelope glycoprotein $\mathrm{gB}$ of HCMV and TLR2 have been described [11]. Binding to the receptor, induced secretion of proinflammatory cytokines but did not take part in the activation of IFN- $\alpha / \beta$ secretion by infected cells [12], suggesting that TLR2 activation by HCMV could not benefit the host. Interestingly, it is known that TLR are also involved in the release of anti-inflammatory cytokines and that TLR2 ligation could be a strong mediator of anti-inflammatory effects, including the secretion of IL-10, IL-4 and activation of regulatory T cells (Treg) (for review see [13]). We may then assume that triggering of TLR2 by HCMV on
DCs could provide the virus with an additional trick to resist the host defence through activation of anti-inflammatory mechanisms. Besides this putative TLR2-mediated immunosuppression, an additional escape mechanism could be considered due to the ability of HCMV to bind to DC-SIGN on dendritic cells, another PRR known to mediate suppression of DCs function in models of bacterial infections [14]. Altogether, these hypotheses remain to be sustained by experiments on DCs either by the myeloid or plasmacytoid subset.

CD8+ T cell response and HCMV immunoevasins (Fig. 1)

Cytotoxic CD8+ T cells are the main effectors involved in the clearing of HCMV-infected targets and it was not surprising that during its evolution with the host, the virus ensured its protection against CTL by encoding the proteins, which were able to disrupt the major histocompatibility complex (MHC) class I antigen processing pathway. Numerous viral gene products have been identified with the ability to impair the molecular cascade leading to the transport of MHC-I-peptide complexes from the endoplasmic reticulum (ER) to the surface of antigen presenting cells. The US2 to US11 region of the genome, encodes proteins (US2 and US11), which translocate the newly synthesized MHC class I heavy chains from ER to the cytosol for their degradation by proteasomal enzymes. Through its binding to the transporter associated with antigen processing (TAP), the US6 gene product blocks the translocation of peptides from the cytosol to ER thus impairing their association with MHC class I heavy chains. Finally, even though some MHC-I-peptide complexes could escape, the US3 protein would cause their retention in the ER, thereby avoiding their transport to the cell surface (reviewed in [6]). Providing that all these escape mechanisms are able to block antigen presentation in infected DCs, we may ask how the host could mount a $\mathrm{CD} 8+\mathrm{T}$ cell response against HCMV following primary infection, and how these effectors could be efficient in controlling re-infection or virus reactivation in latently infected healthy individuals? We can first assume that efficiency of immunoevasins could be lower in vivo than in vitro and even in some cell types compared to others. Indeed, besides their locus-preference abilities to target class I heavy chains of HLA-A and $-\mathrm{B}$ but not $-\mathrm{C}$ and $-\mathrm{G}$ [15], US2 and US11 proteins were shown to exhibit different efficiencies in dendritic cells compared with fibroblasts and astrocytoma cells [16]. The notion that, even though they are produced very early after infection, immunoevasins could be less efficient in vivo than expected from in vitro studies is supported by recent findings demonstrating a broad repertoire of $\mathrm{CD} 8+\mathrm{T}$ cells with specificities against IE, E and L proteins. 


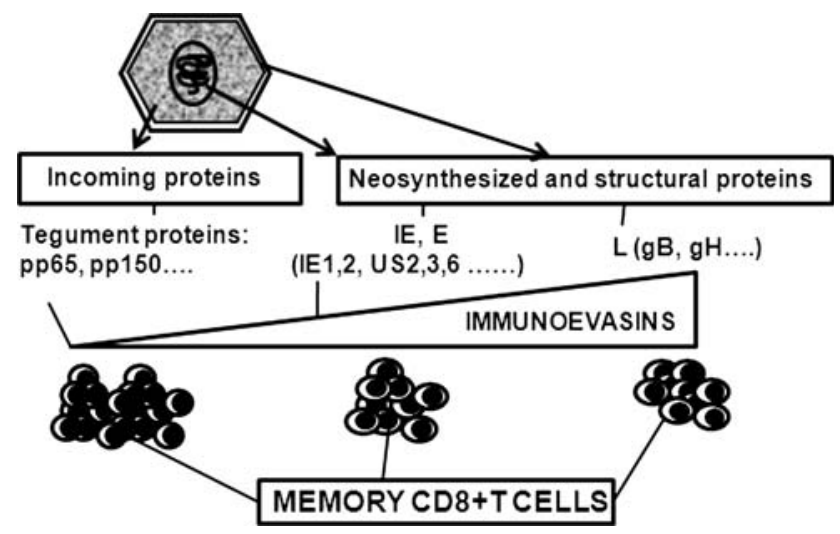

Fig. 1 CD8+ T cell response and immunoevasins. Despite expression of numerous immunoevasins throughout the course of infection, memory $\mathrm{CD} 8+\mathrm{T}$ cells against IE, $\mathrm{E}$ and $\mathrm{L}$ antigens are found in circulating blood of HCMV-seropositive donors

\section{T cell repertoire against HCMV exhibits a broad pattern of specificities despite expression of immunoevasins: possible explanations}

To examine the frequency and specificity of CD8+ CTL, a recent study using fibroblasts as antigens presenting cells infected with a mutant strain deleted of the US immunoevasins, showed that most effector T cells were directed against IE and $\mathrm{E}$ antigens [17]. These data suggest that after primary infection in vivo, naïve $T$ cells could be activated by DCs which acquired IE and E proteins available before the time point at which US immunoevasins are expressed in amounts sufficient to exert their blocking activities. We can also assume that proteins contained in the viral tegument like pp65 and pp150, as well as proteins encoded by viral RNA entrapped during assembly of new virions, could be processed into the MHC-I proteasomal machinery, immediately after adsorption of the virus to the cell surface. Presentation of input pp65 to effector memory CD8+ T cells in vitro has been demonstrated after infection of monocytesderived myeloid DCs with either the laboratory strain AD169 [18] or VHLE (personal data), a clinical isolate of HCMV, demonstrating that endogenous viral gene expression was not required. We further showed that incoming pp65 could be processed and presented to CD8+ T cells by non-professional antigen presenting cells such as astrocytoma cell line U373MG, infected with AD169 for $6 \mathrm{~h}$ [19]. Even though direct presentation of input proteins by DCs in vitro required high multiplicity of infection, we have no information on requirements to make this process efficient in vivo, and moreover, to ensure priming of naïve $T$ cells.

An extensive analysis of $\mathrm{CD} 8+\mathrm{T}$ cell responses against HCMV in seropositive blood donors, by using an IFN- $\gamma$ ELISPOT assay with synthetic peptides, revealed a broad repertoire of viral antigens encoded in the immediate-early, early, and late phases of replication, even including structural proteins like $\mathrm{gB}$ [20]. Within the numerous antigens recognized by memory CD8+ T cells of healthy HCMVseropositive donors tested with various HLA haplotypes, IE1 and pp65 were the most immunodominant, confirming observations claimed by several groups for many years. Moreover, CTL detected in young infants with HCMV infection were directed at both IE1 and pp65 and their appearance correlated with decrease in viral load [21, 22]. This supports the notion that immunodominance could be governed by antigenic dominance, reflecting antigen amounts available at the right time when immunoevasins are not yet effective. Overall it seems that the immunodominance hierarchy in primary response against HCMV could be related to timing of viral antigen expressed in DCs and present in their vicinity. This was corroborated by a more recent study intended to screen the immunogenicity of 213 HCMV open reading frames (ORF), using cytokine flow cytometry and overlapping 15-mer peptides [23]. This study demonstrated the ability of peptides from 151 ORF to induce activation of CD4+ and CD8+ T cells from HCMVseropositive donors with various MHC haplotypes, providing additional proof that the spectrum of memory $\mathrm{T}$ cells against the virus is broader than expected. We may then ask whether the kinetics of viral replication at sites of virus entry could be connected to induction of responses against such a broad spectrum of IE, E and $\mathrm{L}$ antigens.

We can make the assumption that in tissue cells which are targets of primary infection, for instance in mucosa, a productive lytic cycle could be shortly achieved so that soluble as well as cell-associated antigens could be available for internalization by DCs. On the other hand, we can consider that during this primary phase, new virions could be formed and bind to still uninfected cells. Then, CD8+ T cells directed against incoming tegument proteins and IE antigens and which acquired their effector function in the early days following virus entry, could kill these new targets, thereby providing an additional source of antigens for cross-presentation by DCs (Fig. 2, I). These assumptions require further in vivo experiments for instance in the MCMV model.

\section{Role of cross-presentation to bypass viral subversion and in moulding $\mathrm{T}$ cell repertoire}

To further support the hypothesis that immunodominance hierarchy and broadness of $\mathrm{T}$ cell repertoire could correlate with the presence of high dose antigens as well as with antigens released by destruction of infected cells, we suggested that DCs could play a major role in both sensing and killing of infected cells in their vicinity. Since infected DCs could be paralysed [4] and made unable to ensure activation of 


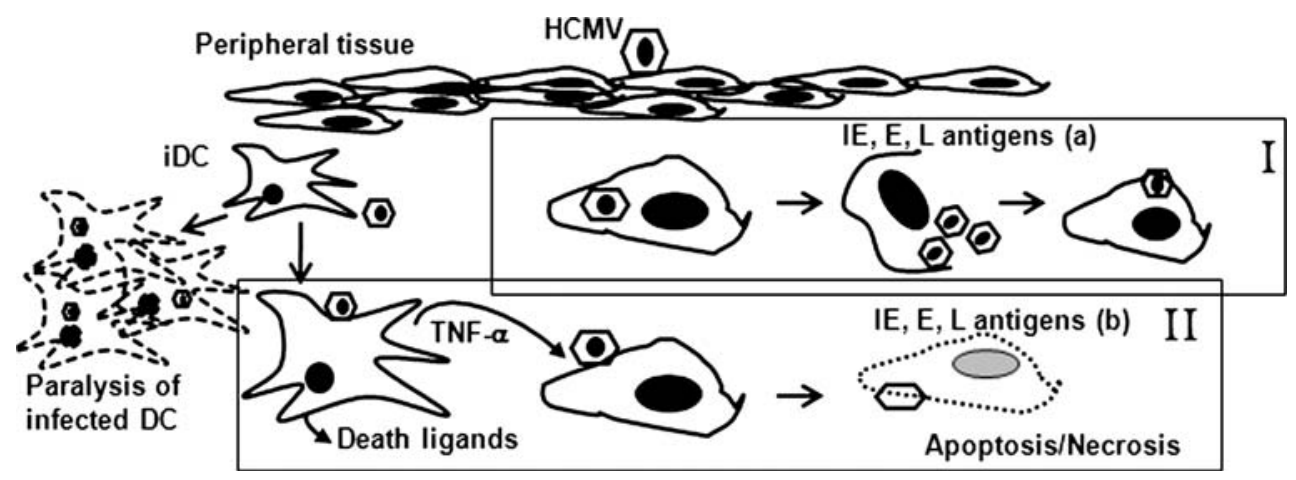

Fig. 2 Hypothesis to explain the extent of $\mathrm{T}$ cell repertoire against HCMV antigens. I Following lytic cycle, IE, E and L proteins could be released and serve as a source of antigens for dendritic cells. Moreover, newly produced virions could bind to still uninfected cells. II Activation of dendritic cells through adsorption of the virus induces secretion

naive $\mathrm{T}$ cells directed against viral antigens, we suggested a scenario where uninfected DCs could acquire viral antigens for cross-presentation to CD8+ T lymphocytes. In an in vitro model where HCMV-infected fibroblasts were co-cultured with monocyte-derived dendritic cells, we demonstrated that immature DCs (iDC) acquired a mature phenotype ( $\mathrm{mDC})$, as assessed by an up-regulation of CD80, CD86 and CD83 expression on the cell surface and by the secretion of huge amounts of TNF- $\alpha[24,25]$. We further demonstrated that HCMV-infected fibroblasts were sensitive to apoptosis mediated by TNF- $\alpha$, but only within the first hours after addition of the virus. Indeed, at later stages after infection, cells are protected by UL36 and UL37 anti-apoptotic functions acting on the death initiating signaling complex (DISC) and mitochondria, respectively $[26,27]$. We then argued in favour of a scenario where stimulated DCs acquired the ability to kill adjacent infected cells through secretion of death ligands such as TNF- $\alpha$ (Fig. 2, II) as we recently demonstrated in a co-culture model where iDC were added to infected fibroblasts in the presence of a pan-caspase inhibitor. We further demonstrated that in these conditions DCs could internalize pp65 associated with early apoptotic and necrotic fibroblasts for processing and cross-presentation to $\mathrm{CD} 8+\mathrm{T}$ cells but with less efficiency when fibroblasts were treated with inhibitors of caspases [19,25]. Indeed, ingestion of dead cells by DCs is thought to provide one of the strongest activation signals for cross-presentation to CD8+ T cells and we observed that resistance of fibroblasts to apoptosis in later stages of infection contributed to reduce the efficiency of cross-presentation [25]. Proposed scenarios for cross-presentation have been recently reviewed [28] and are depicted in Fig. 3. The first scenario is based on the export of antigens from endocytic/phagocytic vesicles to the cytosol allowing their delivery into the direct MHC class I pathway where they are processed into peptides by the same proteasomal degra- of death ligands including TNF- $\alpha$ [25] leading to killing of infected cells in their vicinity. Then, immature but uninfected dendritic cells could internalize soluble $(a)$ as well as cell-associated $(b)$ IE, E and L antigens

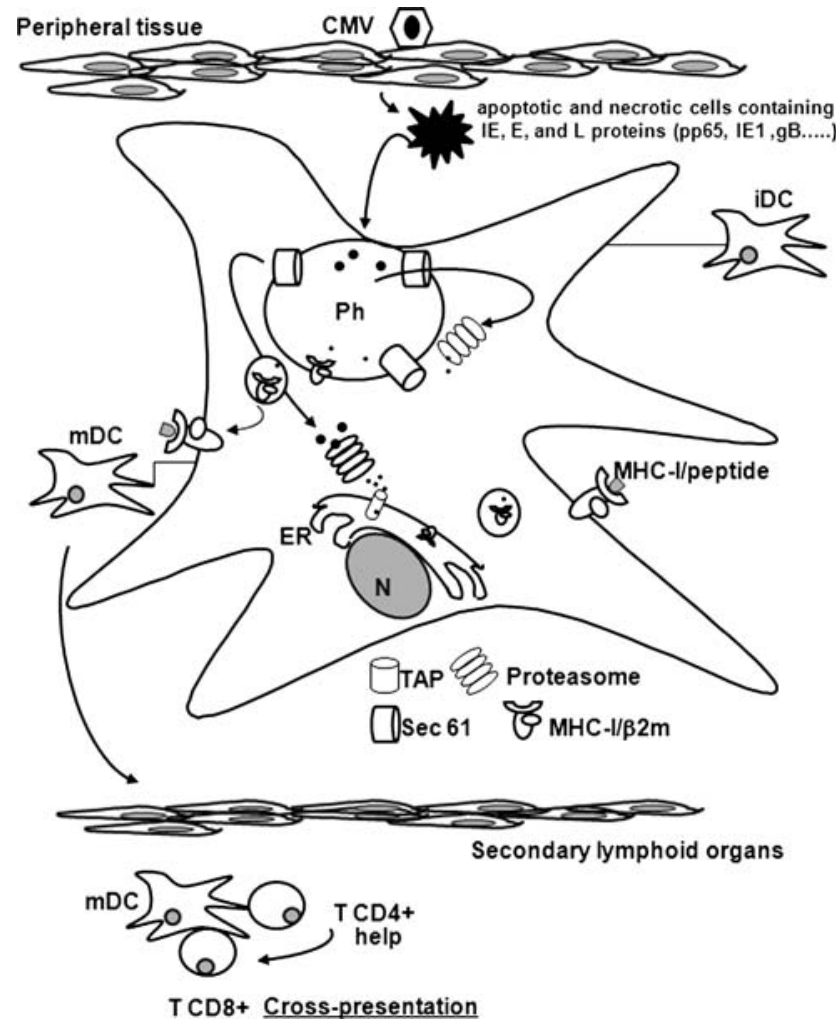

Fig. 3 Cross-presentation of HCMV antigens by dendritic cells to CD8+ T cells. Phagocytosis of apoptotic and necrotic cells containing IE, E and L proteins by immature dendritic cells (iDC), leads to processing of the antigens (closed circles) either within autonomous phagosomes $(\mathrm{Ph})$ or within the ER, following translocation from the phagosome through translocon Sec61. Dendritic cells which then acquired a mature phenotype ( $\mathrm{mDC}$ ) migrate to secondary lymphoid organs where activation of $\mathrm{HCMV}$-specific naïve $\mathrm{CD} 4+$ and $\mathrm{CD} 8+\mathrm{T}$ cells takes place. $\mathrm{N}$, nucleus

dation pathway that is used for endogenously produced antigens. In the second scenario, phagocytosis of particulate antigens initiates the fusion with endoplasmic reticulum membranes and gives rise to a novel organelle with 
autonomous processing and presenting capacities. Processed antigens are then exported to the cytosol by a translocation channel Sec61, coated by the ubiquitinating complex and recaptured by the phagosome-associated proteasomal machinery. Peptides with proper epitope structure are then retro-translocated by TAP molecules into the phagosome where they are loaded onto the MHC-I- $\beta_{2}$-microglobulin dimers before being recycled back to the cell membrane. Interestingly, when DCs were added to lateinfected fibroblasts their maturation was inhibited under the effect of cytokines such as TGF- $\beta$ and of the virokine UL111a (cmvIL-10) secreted by infected fibroblasts, and their ability to induce activation of anti-pp65 CD8+ T cells was impaired [24, 25]. At a time where fibroblasts are sensitive to dendritic cell-induced apoptosis, several viral antigens are available for cross-presentation, including tegument and envelope proteins such as pp65, pp150, pp28, $\mathrm{gB}, \mathrm{gH}$ derived from the input virus, either associated with dead cells or in soluble forms. Neosynthesized IE and E proteins could be as well available at this time point. Accordingly, it could be expected that in primary response, naive CD8+ T cells directed against these IE, $\mathrm{E}$ and $\mathrm{L}$ viral antigens could be activated, providing an explanation for the presence of a broad repertoire of memory $\mathrm{T}$ cells in HCMV-seropositive donors. The immunodominance of some viral antigens may also reflect their relative expression level at a time when they could be internalized by DCs and before the immunosuppressive effect of TGF- $\beta$ and cmvIL-10. Then, shape and size of the memory T cell repertoire could correlate with the nature and amount of viral antigens cross-presented by DCs, but patterns of effector T cells frequencies in HCMV-seropositive donors, as recently assessed [23], could reflect expansion of $\mathrm{T}$ cells against recall antigens following re-infection and/or reactivation. History of recall infections and reactivation in a given donor may explain reversal in the immunodominance hierarchy which may be influenced by differential antigen presentation during primary and secondary responses supposed to involve dendritic and non-dendritic cells, respectively. Analysis of $\mathrm{T}$ cell response in transplanted patients who did not develop HCMV disease showed contrasting results. Indeed, high frequencies of IE1- but not pp65-specific CD8+ T cells were found protective in one case [29] whereas, rising numbers of pp65-specific T cells correlated with immune reconstitution in another case [30]. Interestingly, all patients protected by IE1-specifc $\mathrm{T}$ cells were HCMV-seropositive before transplantation suggesting that viral reactivation was responsible for expansion of CTL against IE1 which is produced in large amounts in the IE phase. In contrast, all patients with high counts of pp65specific CTL received a graft from HCMV-seropositive donors suggesting that re-infection could be responsible for expansion of CTL directed against incoming pp65. These results suggest once more that the expansion of virus-specifc CD8+ T cells in secondary responses could depend on the timing of antigen expression and could be regulated by cross-competition favoring $\mathrm{T}$ cells that are able to rapidly detect infected cells as demonstrated recently in a mouse model of Poxvirus infection [31].

In conclusion, interplay between dendritic cells and HCMV in primary infection may favour activation of naive $\mathrm{T}$ cells with specificity for various viral antigens provided that they have been captured by uninfected DCs but their ability to proliferate and to control virus spreading in secondary responses may depend on conditions under which re-infection and reactivation occur in the host. Relevance of cross-presentation in $\mathrm{T}$ cell response against viral infections was recently highlighted by data reporting the ability of plasmacytoid dendritic cells (pDCs) to cross-present HIVderived antigens to CD8+ T cells [32]. A better knowledge of cross-presentation function by myeloid and plasmacytoid DCs in shaping $\mathrm{T}$ cell response against HCMV antigens may help to improve vaccine design and cellular immunotherapy.

Acknowledgments Grants were from INSERM (Institut National de la Santé et de la Recherche Médicale), ARC (Association pour la Recherche sur le Cancer) and Midi-Pyrénées region.

\section{References}

1. Geijtenbeek TB, Kwon DS, Torensma R, van Vliet SJ, van Duijnhoven GC, Middel J, Cornelissen IL, Nottet HS, KewalRamani VN, Littman DR, Figdor CG, van Kooyk Y (2000) DC-SIGN, a dendritic cell-specific HIV-1-binding protein that enhances transinfection of T cells. Cell 100:587-97

2. Halary F, Amara A, Lortat-Jacob H, Messerle M, Delaunay T, Houles C, Fieschi F, Arenzana-Seisdedos F, Moreau JF, DechanetMerville J (2002) Human cytomegalovirus binding to DC-SIGN is required for dendritic cell infection and target cell trans-infection. Immunity 17:653-64

3. Hahn G, Jores R, Mocarski ES (1998) Cytomegalovirus remains latent in a common precursor of dendritic and myeloid cells. Proc Natl Acad Sci USA 95:3937-42

4. Beck K, Meyer-Konig U, Weidmann M, Nern C, Hufert FT (2003) Human cytomegalovirus impairs dendritic cell function: a novel mechanism of human cytomegalovirus immune escape. Eur J Immunol 33:1528-38

5. Reeves MB, MacAry PA, Lehner PJ, Sissons JG, Sinclair JH (2005) Latency, chromatin remodeling, and reactivation of human cytomegalovirus in the dendritic cells of healthy carriers. Proc Natl Acad Sci USA 102:4140-5

6. Reddehase MJ (2002) Antigens and immunoevasins: opponents in cytomegalovirus immune surveillance. Nat Rev Immunol 2:831-44

7. Reddehase MJ, Weiland F, Munch K, Jonjic S, Luske A, Koszinowski UH (1985) Interstitial murine cytomegalovirus pneumonia after irradiation: characterization of cells that limit viral replication during established infection of the lungs. J Virol 55:264-73

8. Riddell SR, Watanabe KS, Goodrich JM, Li CR, Agha ME, Greenberg PD (1992) Restoration of viral immunity in immunodeficient humans by the adoptive transfer of T cell clones. Science 257:238-41 
9. Kapp M, Tan S, Einsele H, Grigoleit G (2007) Adoptive immunotherapy of HCMV infection. Cytotherapy 1-13

10. Barton GM (2007) Viral recognition by Toll-like receptors. Semin Immunol 19:33-40

11. Boehme KW, Guerrero M, Compton T (2006) Human cytomegalovirus envelope glycoproteins $\mathrm{B}$ and $\mathrm{H}$ are necessary for TLR2 activation in permissive cells. J Immunol 177:7094-102

12. Boehme KW, Compton T (2006) Virus entry and activation of innate immunity. In: J RM (ed) Cytomegaloviruses molecular biology and immunology. Caister Academic Press, Norfolk, pp 111-130

13. Netea MG, Van der Meer JW, Kullberg BJ (2004) Toll-like receptors as an escape mechanism from the host defense. Trends Microbiol 12:484-8

14. Geijtenbeek TB, Van Vliet SJ, Koppel EA, Sanchez-Hernandez M, Vandenbroucke-Grauls CM, Appelmelk B, Van Kooyk Y (2003) Mycobacteria target DC-SIGN to suppress dendritic cell function. J Exp Med 197:7-17

15. Schust DJ, Tortorella D, Seebach J, Phan C, Ploegh HL (1998) Trophoblast class I major histocompatibility complex (MHC) products are resistant to rapid degradation imposed by the human cytomegalovirus (HCMV) gene products US2 and US11. J Exp Med 188:497-503

16. Rehm A, Engelsberg A, Tortorella D, Korner IJ, Lehmann I, Ploegh HL, Hopken UE (2002) Human cytomegalovirus gene products US2 and US11 differ in their ability to attack major histocompatibility class I heavy chains in dendritic cells. J Virol 76:5043-50

17. Manley TJ, Luy L, Jones T, Boeckh M, Mutimer H, Riddell SR (2004) Immune evasion proteins of human cytomegalovirus do not prevent a diverse $\mathrm{CD} 8+$ cytotoxic $\mathrm{T}$-cell response in natural infection. Blood 104:1075-82

18. Cho HI, Han H, Kim CC, Kim TG (2001) Generation of cytotoxic $\mathrm{T}$ lymphocytes specific for human cytomegalovirus using dendritic cells in vitro. J Immunother 24:242-9

19. Arrode G, Boccaccio C, Lule J, Allart S, Moinard N, Abastado JP, Alam A, Davrinche C (2000) Incoming human cytomegalovirus pp65 (UL83) contained in apoptotic infected fibroblasts is crosspresented to CD8(+) T cells by dendritic cells. J Virol 74:10018-24

20. Elkington R, Walker S, Crough T, Menzies M, Tellam J, Bharadwaj M, Khanna R (2003) Ex vivo profiling of CD8+-T-cell responses to human cytomegalovirus reveals broad and multispecific reactivities in healthy virus carriers. J Virol 77:5226-40

21. Gibson L, Dooley S, Trzmielina S, Somasundaran M, Fisher D, Revello MG, Luzuriaga K (2007) Cytomegalovirus (CMV) IE1and pp65-specific CD8+ T cell responses broaden over time after primary CMV infection in infants. J Infect Dis 195:1789-98

22. Gibson L, Piccinini G, Lilleri D, Revello MG, Wang Z, Markel S, Diamond DJ, Luzuriaga K (2004) Human cytomegalovirus proteins pp65 and immediate early protein 1 are common targets for CD8+ T cell responses in children with congenital or postnatal human cytomegalovirus infection. J Immunol 172:2256-64

23. Sylwester AW, Mitchell BL, Edgar JB, Taormina C, Pelte C, Ruchti F, Sleath PR, Grabstein KH, Hosken NA, Kern F, Nelson JA, Picker LJ (2005) Broadly targeted human cytomegalovirusspecific CD4+ and CD8+ T cells dominate the memory compartments of exposed subjects. J Exp Med 202:673-85

24. Arrode G, Boccaccio C, Abastado JP, Davrinche C (2002) Crosspresentation of human cytomegalovirus pp65 (UL83) to CD8(+) T Cells is regulated by Virus-Induced, soluble-mediator-dependent maturation of dendritic cells. J Virol 76:142-150

25. Mandron M, Martin H, Bonjean B, Lulé J, Tartour E, Davrinche C (2008) Dendritic cell-induced apoptosis of human cytomegalovirus-infected fibroblasts promotes cross-presentation of pp65 to CD8+ T cells. J Gen Virol 89:78-86

26. Skaletskaya A, Bartle LM, Chittenden T, McCormick AL, Mocarski ES, Goldmacher VS (2001) A cytomegalovirus-encoded inhibitor of apoptosis that suppresses caspase- 8 activation. Proc Natl Acad Sci USA 98:7829-34

27. Goldmacher VS, Bartle LM, Skaletskaya A, Dionne CA, Kedersha NL, Vater CA, Han JW, Lutz RJ, Watanabe S, Cahir McFarland ED, Kieff ED, Mocarski ES, Chittenden T (1999) A cytomegalovirus-encoded mitochondria-localized inhibitor of apoptosis structurally unrelated to Bcl-2. Proc Natl Acad Sci USA 96:12536-41

28. Jensen PE (2007) Recent advances in antigen processing and presentation. Nat Immunol 8:1041-8

29. Bunde T, Kirchner A, Hoffmeister B, Habedank D, Hetzer R, Cherepnev G, Proesch S, Reinke P, Volk HD, Lehmkuhl H, Kern F (2005) Protection from cytomegalovirus after transplantation is correlated with immediate early 1-specific CD8 T cells. J Exp Med 201:1031-6

30. Hebart H, Daginik S, Stevanovic S, Grigoleit U, Dobler A, Baur M, Rauser G, Sinzger C, Jahn G, Loeffler J, Kanz L, Rammensee HG, Einsele H (2002) Sensitive detection of human cytomegalovirus peptide-specific cytotoxic T-lymphocyte responses by interferon-gamma-enzyme-linked immunospot assay and flow cytometry in healthy individuals and in patients after allogeneic stem cell transplantation. Blood 99:3830-7

31. Kastenmuller W, Gasteiger G, Gronau JH, Baier R, Ljapoci R, Busch DH, Drexler I (2007) Cross-competition of CD8+ T cells shapes the immunodominance hierarchy during boost vaccination. J Exp Med 204:2187-98

32. Hoeffel G, Ripoche AC, Matheoud D, Nascimbeni M, Escriou N, Lebon P, Heshmati F, Guillet JG, Gannage M, Caillat-Zucman S, Casartelli N, Schwartz O, De la Salle H, Hanau D, Hosmalin A, Maranon C (2007) Antigen crosspresentation by human plasmacytoid dendritic cells. Immunity 27:481-92 